\section{Effect of Chloramphenicol on Ribonucleic Acid Metabolism in E. coli infected with T2 Phage}

IT has been suggested ${ }^{1}$ that the T2-phage infection first induces the formation of new protein in bacteria before the appearance of phage deoxyribonucleic acid and phage-antigenic protein. Quite recently, however, we have made the important additional discovery that the phage infection also induces a synthesis of new ribonucleic acid, or at least a specific change in its metabolism, and that the formation of this special ribonucleic acid is independent of the prior formation of new protein. These facts suggest that the $T 2$-infection first induces the synthesis of new protein via the formation of new ribonucleic acid or the metabolism of this acid.

By adding phosphorus-32 to the medium or by diluting phosphorus-32 in the medium with nonradioactive phosphorus at various times after $T 2$ infection, we found that phosphorus-32 was rapidly incorporated into and rapidly liberated from ribonucleic acid for a long time after infection, when the net ribonucleic acid synthesis had almost stopped. On the other hand, the ribonucleic acid-phosphorus in uninfected coli was reported to be metabolically inactive ${ }^{2}$. It was also found that the ribonucleic acid labelled with phosphorus-32 during the first $15 \mathrm{~min}$. after $T 2$-infection was different in mononucleotide composition from that of uninfected coli, confirming the results of Volkin et $a .^{3}$ (see Table 1). Thus the ribonucleic acid formed after $T 2$-infection is distinct from that of uninfected host coli both in chemical composition and metabolic activity, and is therefore considered to be specific for $T 2$-infection.

Table 1. PHOSPHORUS INCORPORATION INTO RIBONUCLEIC ACID MONONUCLEOTIDES OF $E$. coli $B(A-20)$ AFTER T2-INFECTION*.

\begin{tabular}{|c|c|c|c|c|}
\hline & Guanylic & Adenylic & Cytidylic & Uridylic \\
\hline Uninfected control & 1.2, & 1.00 & $1.0_{2}$ & 0.90 \\
After T2-infection & 0.80 & 1.00 & 0.64 & 0.98 \\
After T2-infection & 0.89 & 1.00 & 0.66 & 0.89 \\
$\begin{array}{c}\text { Chloramphenicol } \\
\text { (50 } / / \text { ml.) added } \\
\text { before infection }\end{array}$ & & & & \\
\hline
\end{tabular}

* Phosphorus-32 was fed during the first 15 min. after $T 2$-infection, and the ribonucleic acid fraction obtained was hydrolysed by alkali and analysed by paper electrophoresis. The details of this experiment will be published elsewhere.

We then studied the effect of chloramphenicol on the ribonucleic acid metabolism in $T 2$-infected coli. It was found that chloramphenicol, when added shortly before $T 2$-infection, blocked the formation of protein and deoxyribonucleic acid, but not the ribonucleic acid synthesis after infection. The ribonucleic acid synthesis after infection seemed to be rather stimulated by the addition of chloramphenicol. This ribonucleic acid, which is formed without the prior formation of protein after T2-infection, is also characteristic of $T^{\prime} 2$-infection in its mononucleotide composition (see Table 1). These results, which show that the $T 2$-infection induces a synthesis of new ribonucleic acid or at least a specific change in its metabolism without the prior synthesis of new protein, are in great contrast with the findings that new deoxyribonucleic acid synthesis requires the prior formation of new protein after $T 2$-infection ${ }^{4,5}$.

The stimulation of the ribonucleic acid synthesis in infected coli was more easily found when chloramphenicol was added at $10 \mathrm{~min}$. after infection. It was shown that the addition of chloramphenicol inhibited the liberation of phosphorus from the ribonucleic acid formed after infection and resulted in the stimulation of its net synthesis. There should be, therefore, a correlation between the protein synthesis and the phosphorus liberation from ribonucleic acid. The deoxyribonucleic acid synthesis in infected coli was not inhibited by the addition of chloramphenicol at $10 \mathrm{~min}$. after infection, as Tomizawa et al. found ${ }^{4}$, and there seems no apparent relation between deoxyribonucleic acid synthesis and ribonucleic acid breakdown in infected coli, although it is probable that a part of the phosphorus in the former can be derived from the latter. We have not yet been able to obtain protein synthesis without ribonucleic acid formation after $T 2$-infection.

This work was supported in part by a grant from the Scientific Research Fund of the Ministry of Education of Japan.

ITARU WATANABE

YUKIO KIHO

KIN-ICHIRO MIURA

Institute of Science and Technology,

University of Tokyo.

Dec. 23.

${ }^{1}$ Watanabe, I. , Biochim. Biophys. Acta, 25, 665 (1957).

${ }^{2}$ Hershey, A. D., J. Gen. Physiol., 38, 145 (1954).

${ }^{3}$ Volkin, E., and Astrachan, L., Virology, 2, 149 (1956).

4 Tomizawa, J., and Sunakawa, S., J. Gen. Physiol., 39, 553 (1956).

¿ Burton, K., Biochem. J., 61, 473 (1955).

\section{Changes in Adenine Nucleotides of Squid Muscle}

IT was reported in an earlier communication ${ }^{1}$ that after slow freezing of carp muscle there is a very rapid splitting of adenosine triphosphate accompanied by an instantaneous deamination, as a result of which inosine monophosphate accumulated predominantly. The mechanism of these phenomena is assumed to be that the action of adenosine triphosphatase or adenosine monophosphate deaminase is not affected by such low temperatures, while that of $5^{\prime}$-nucleotide phosphatase is reduced ${ }^{2}$. On the other hand, we have investigated the changes of nucleotides in squid muscle under the same conditions as those described previously and the specific phenomena have been observed to differ from those of carp muscle. This will be described in the present communication.

Fresh squid muscle was kept at $-5^{\circ} \mathrm{C}$. for $50 \mathrm{hr}$. ; during the first $3 \mathrm{hr}$. the muscle was frozen completely. $2 \mathrm{gm}$. of frozen muscle were ground, by hand in a mortar, in $20 \mathrm{ml}$. 4 per cent perchloric acid solution. The extract was filtered, and the filtrate was used for analysis.

Adenine and hypoxanthine nucleosides and nucleotides were separated by the hydrochlorie acid gradient. elution system on 'Amberlite' IRA-400. Inosine and hypoxanthine were separated by paper chromatography. The details of the system ${ }^{3}$ and the methods of paper chromatography ${ }^{4}$ have already been. described.

In Fig. 1 the concentrations of the compounds. present after various time-intervals are shown. It can be seen that during the first $10 \mathrm{hr}$. a rapid dephosphorylation of adenosine triphosphate occurred. with a concomitant accumulation of the diphosphate and of the monophosphate; furthermore, the monophosphate accumulated gradually during. $25 \mathrm{hr}$. from the start, while the diphosphate de. creased. Adenosine monophosphate thus formed, 